\title{
Prevalence of Inducible Clindamycin Resistance among Nasal Carriage Staphylococcus aureus among Healthy Population
}

\author{
Manish Kumar Diwakar ${ }^{1}$, Ankur Goyal ${ }^{2} *$, Santosh Verma $^{3}$ and Neha Srivastava ${ }^{4}$ \\ ${ }^{1}$ Department of Microbiology, MLB Medical College, Jhansi (UP), India \\ ${ }^{2}$ Department of Microbiology, SN Medical college, Agra, India \\ ${ }^{3}$ Department of Community Medicine, MLB Medical College, Jhansi (UP), India \\ ${ }^{4}$ Department of Microbiology, Rama Medical College, Kanpur (UP), India \\ *Corresponding author
}

\begin{tabular}{l} 
Ke y w o r d s \\
$\begin{array}{l}\text { Staphylococcus aureus, } \\
\text { MRSA, Nasal carriage, } \\
\text { Inducible clindamycin } \\
\text { resistance, D-test }\end{array}$ \\
\hline Article Info \\
\hline $\begin{array}{l}\text { Accepted: } \\
18 \text { January } 2018 \\
\text { Available Online: } \\
10 \text { May } 2018\end{array}$ \\
\hline
\end{tabular}

The increasing resistance to many antimicrobial agents is seen in Staphylococcus aureus in all healthcare facilities. The commonest antibiotic for the treatment of the Methicillin and multi drug resistant Staphylococcal infections is clindamycin. Emergence of these resistances in Staphylococcus aureus has resulted in failure of clindamycin therapy. A total 220 Nasal swabs from healthy persons were collected and processed by standard microbiological procedures. Staphylococcus aureus was isolated in 48 cases $(21.82 \%)$ among which $6.25 \%$ were Methicillin resistance. All the Staphylococcus aureus isolates were sensitive to Amikacin, mupiriocin, teicoplanin, vancomycin and linezolid (100\%). Ampicillin was the most resistant (54\%) antibiotic followed by Erythromycin (40\%), ciprofloxacin (33\%), cotrimoxazole (31\%), Ampicillin calvulanic acid $(23 \%)$, clindamycin $(17 \%)$ and chloramphenicol $(15 \%)$, gentamycin (10\%), levofloxacin (8\%) and doxycycline (2\%). Inducible clindamycin resistance among $S$. aureus isolates was $6.25 \%$. The percentage of inducible clindamycin resistance was higher (66.67\%) among MRSA compared to MSSA (2.23\%). The D-test is a simple and effective tool for the phenotypic detection of inducible clindamycin resistance in $S$. aureus. It should be taking forward in routine practice for proper patient management and empirical therapy guideline.

\section{Introduction}

Staphylococcus aureus and coagulase negative Staphylococcus (CONS) are well known causative agent for a various infections which range from minor skin and soft tissue infections to life threatening conditions such as septicaemia, endocarditic and pneumonia (Lowy FD, 1998). The emergence of drug resistance among Staphylococci is a global threat. (Fiebelkorn et al., 2013) Methicillin resistant S. aureus (MRSA) is an established nosocomial pathogen and its rate has drastically increased in the recent years (Schreckenberger et al., 2004). Clindamycin, an antimicrobial agent which belong to the macrolide-lincosamide-streptogramin B (MLSB) family, is frequently used for treatment of infections caused by Staphylococcus aureus (S. aureus) as well as 
MRSA. Clindamycin is the preferred agent due to its excellent pharmacokinetic properties.

However, the widespread use of the MLSB family of antimicrobials has led to the emergence of resistance to MLSB antibiotics (Fahriye Efgan et al., 2011).

The macrolide-lincosamide-streptogramin B (MLSB) antibiotics are structurally unrelated but they have similar mode of action. They act by inhibiting the bacterial protein synthesis by binding to the 23S rRNA of the 50S ribosomal subunit (Ciraj AM et al., 2009; Roberts et al., 1999a, b).

The antimicrobial resistance to the MLSB group may be caused by different mechanisms which include the macrolide efflux pump, target site modification and enzymatic antibiotic inactivation. The modification of the ribosomal target is encoded by a multiallele plasmid coded erythromycin ribosome methylase (erm) gene which is responsible for production of the methylase enzymes. These enzymes cause methylation of the A2058 residue which is located in the conserved domain $\mathrm{V}$ of the $23 \mathrm{~S}$ rRNA component of the $50 \mathrm{~S}$ ribosomal subunit, which leads to cross resistance and called as the MLSB - resistant phenotype (Weisblum, 1995).

The resistance to the MLSB antibiotics can be either constitutive (MLSBc) or inducible (MLSBi) type. If the erm genes are consistently expressed, the organisms may show resistance to erythromycin (ER), clindamycin $(\mathrm{CD})$ and to other members of the MLSB group and the organism are said to be of the MLSBc phenotype. However, if the erm genes require an inducing agent to express the resistance to Clindamycin (CD), then the organisms are said to be of the MLSBi phenotype.
MLSBi phenotype is resistant to Erythromycin and sensitive to Clindamycin in vitro.

The Clindamycin therapy in such patients don't respond which lead to clinical failures [9-12]. Low level of Erythromycin is an inducer of the MLSBi phenotype and this is the basis of the D-test (Weisblum B, 1973).

Staphylococci can also develop macrolide resistance through efflux pump encoded by the macrolide streptogramin resistance (msrA) gene, leading to resistance to the macrolides and the type B streptogramins, but not to the lincosamides. These isolates are known as MS phenotype and they show in vitro resistance to Erythromycin and susceptibility to Clindamycin. In this condition Clindamycin therapy responds well. Therefore, differentiating these two mechanisms of resistance would prevent the clinical failure of the therapy.

The Clinical Laboratory Standards Institute (CLSI) has recommended the ER-CD disc approximation test (D-zone test) to detect the inducible clindamycin resistance (CLSI guideline, 2017).

There is no study about clindamycin resistance in nasal carriage in this region. This study was therefore taken up to close this gap in our knowledge. The aim of the present study was to detect the prevalence of inducible clindamycin resistance among the nasal isolates of Staphylococci.

Anterior nares is the ecological niches of the $S$. aureus which make the organism a potent agent for nosocomial infection as well as community associated staphylococcal infection. The carriers of MRSA may transmit the pathogen to patients and community leading to various complications associated with MRSA infections like pneumonia and septicaemia, etc (Lowy, 1998) Thus, the 
prevalence of inducible clindamycin resistance in $S$. aureus isolated from anterior nasal cavity was evaluated.

\section{Materials and Methods}

The study was conducted in the Department of Microbiology, Govt. MLB Medical College, Jhansi (UP) from January 2016 to July 2017. A total of 220 nasal swabs from normal healthy persons were collected in this study. None of the individual had a history of illness or treatment with an antibiotic in the last 6 months. Nasal swabs were collected from all the participants by using sterile cotton swabs which was soaked in sterile normal saline, by rotating the swabs in both the anterior nares consecutively. The swabs were processed immediately by inoculating the samples from them onto blood agar plates. The plates were incubated aerobically at $37^{\circ} \mathrm{C}$ for 24 hours. The organism which were isolated were identified on the basis of their colony morphologies and Gram's stain, catalase, coagulase, mannitol fermentation and the DNAase tests by following the standard microbiological techniques (Koneman et al., 2016).

Antimicrobial susceptibility testing of the nasal isolates to Amikacin (30mcg), Ampicillin (10mcg), Ampicillin + calvulanic acid (30/15mcg), Clindamycin (2mcg), Chloramphenicol (30mcg), Co-Trimoxazole (1.25/23.75mcg), Ciprofloxacin (5mcg), levofloxacin (5mcg), mupirocin (5mcg), Doxycyclin (30mcg), Erythromycin $(15 \mathrm{mcg})$, Gentamycin (10mcg), Linezolid (30 mcg), Teicoplanin (30mcg) and Vancomycin (30mcg) was carried out by the standard Kirby Bauer disc diffusion test as per Clinical and Laboratory Standards Institute (CLSI) guidelines. All antibiotic disc was manufactured by HiMedia pvt ltd, Mumbai.

Methicillin resistance was detected by taking cefoxitin as a surrogate marker. MRSA was detected by the cefoxitin disc diffusion test, using a $30 \mu \mathrm{g}$ disc (an inhibition zone diameter of $\leq 21 \mathrm{~mm}$ was reported as oxacillin or methicillin resistant and a zone diameter of $\geq 22 \mathrm{~mm}$ was considered as sensitive) and by growth on oxacillin screen agar, incorporating $4 \% \mathrm{NaCl}$ and $6 \mu \mathrm{g} / \mathrm{ml}$ of oxacillin (HiMedia, Mumbai), as per CLSI guidelines.

The erythromycin and clindamycin discs were placed at a distance of $15 \mathrm{~mm}$ apart edge to edge. After $24 \mathrm{hrs}$ of aerobic incubation at 370C three different phenotypes were interpreted as (Kloos WE et al., 1999):

The constitutive MLSB phenotype (MLSBc): In this phenotype Staphylococcal isolates shows resistant to both Erythromycin (zone of inhibition $\leq 13 \mathrm{~mm}$ ) and Clindamycin $(\leq 14 \mathrm{~mm})$.

The Inducible MLSB Phenotype (MLSBi): In This phenomenon Staphylococcal isolates shows resistance to Erythromycin $(\leq 13 \mathrm{~mm})$ and sensitivity to Clindamycin $(\geq 21 \mathrm{~mm})$ with a D-shaped zone of inhibition around the Clindamycin disc with flattening adjacent side towards erythromycin disc.

The MS phenotype: In This phenotype isolates shows sensitive to Clindamycin $(\geq 21 \mathrm{~mm})$ and resistance to Erythromycin $(\leq 13 \mathrm{~mm})$.

S. aureus BAA 977 and S. aureus BAA 976 were used as the positive and negative controls, respectively for MLSB Antibiotics.

ATCC 43300 and ATCC 25923 were used as positive and negative controls respectively for cefoxitin resistance (Baird et al., 2016; CLSI; 2017)

\section{Results and Discussion}

A total of 220 nasal isolates was collected from 220 normal persons of Bundelkhand region. Among them, $48(21.82 \%)$ were $S$. 
aureus and $172(78.12 \%)$ were coagulase negative Staphylococci. Among the Staphylococcal isolates, $3(6.25 \%)$ were MRSA, 45 (93.75\%) were MSSA (methicillin sensitive S.aureus), 26 (15.12\%) were MRCoNS (methicillin resistant CoNS) and 146 (84.88\%) were MSCoNS (methicillin sensitive CoNS). Erythromycin resistance was seen in $14(29.29 \%)$ isolates while 8 (16.7) isolates found to be resistant to Clindamycin among $S$. aureus isolates. Among these isolates Induciable clindamycin was seen in 3 $(6.3 \%)$ isolates of $S$. aureus. Constitutive MLSB phenotype (MLSBc) and the MS phenotype was seen in $6(12.5 \%)$ and 9 $(18.75 \%)$ isolates among $S$. aureus respectively.

The percentage of inducible clindamycin resistance was higher among methicillinresistant S. aureus (MRSA) (33.3 \%) compared to methicillin-sensitive $S$. aureus (4.4\%). It was observed that Inducible Clindamycin Resistance (MLSBi) isolates were significantly more among the MRSA than the MSSA isolates. Similarly, erythromycin resistance was seen in 65 (37.79\%) of the CoNS isolates. Among these isolates Inducible clindamycin resistance (MLSBi) was seen in 7 (4.07\%) isolates. A total $36(20.9 \%)$ and $29(16.86 \%)$ isolates showed Constitutive MLSB phenotype (MLSBc) and MS phenotype isolates respectively among CONS. (The result is shown in Table 1).

The prevalence of the $S$. aureus nasal carriage was higher among the male $(27 ; 56.25 \%)$ than among the female $(21 ; 43.75 \%)$. All the Staphylococcus aureus isolates were sensitive to Amikacin, mupiriocin, teicoplanin, vancomycin and linezolid (100\%). Ampicillin was the most resistant (54\%) antibiotic followed by Erythromycin (40\%), ciprofloxacin (33\%), cotrimoxazole (31\%), Ampicillin calvulanic acid (23\%), clindamycin (17\%) and chloramphenicol $(15 \%)$, gentamycin $(10 \%)$, levofloxacin $(8 \%)$ and doxycycline $(2 \%)$. Methicillin resistance was seen in $6.25 \%$ of the $S$. aureus isolates, both by the disc diffusion test and by the Oxacillin Screen Agar (OSA) test. Figure 1 shows graphical presentation of antimicrobial resistance percentage of nasal carriage MRSA and MSSA.

The increasing resistance pattern to various antimicrobial agents is observed in Staphylococcus aureus in all healthcare facilities these days. The most notable example is the emergence of Methicillinresistant Staphylococcus aureus (MRSA) and MDR MRSA. The commonest antibiotic for the treatment of the Methicillin and multi drug resistant Staphylococcal infections is clindamycin. It is cost effective too. Emergence of these resistances makes difficult to treat $S$. aureus infections. The empirical treatment for the Staphylococcal infection is now not effective, due to the emergence of multi drug resistant strains, especially MRSA.

The increasing frequency of the Staphylococcal infections and the changing patterns in antimicrobial resistance has led to a renewed interest in the use of the clindamycin for such infections. Clindamycin resistant strains may develop inducible and spontaneous constitutively resistant phenotype. So in vitro and in vivo sensitivity of clindamycin is necessary for such isolates. The inducible clindamycin resistance is usually not detected by the routine antibiotic susceptibility testing and it results in treatment failures. The identification of Staphylococcus aureus and the detection of inducible clindamycin resistance by using a standard microbiological protocol provide effective patient management.

In the present study, a total of 220 nasal isolates was collected from 220 normal 
persons of Bundelkhand region. Among them, (Sharon Rainy et al., 2013; Adithya et al., $48(21.82 \%)$ were S. aureus and $172(78.12 \%) \quad 2009 ; \mathrm{a}, \mathrm{b})$ who found the $22.2 \%$ and $13 \%$ of were coagulase negative Staphylococci. nasal carriage $S$.aureus respectively. However, shobha et al., (2015) don't find any nasal The similar finding was also observed by carriage in the similar study.

Table.1 Resistance pattern of staphylococcal species for erythromycin and clindamycin

\begin{tabular}{|c|c|c|c|c|c|c|}
\hline \multirow[t]{3}{*}{$\begin{array}{l}\text { Resistance } \\
\text { phenotype }\end{array}$} & \multicolumn{3}{|c|}{ S. aureus $\mathrm{n}=48(21.82 \%)$} & \multicolumn{3}{|c|}{$\begin{array}{l}\text { Coagulase negative staphylococci } n=172 \\
(78.12 \%)\end{array}$} \\
\hline & Mrsa & Mssa & \multirow{2}{*}{$\begin{array}{l}\text { total } \\
(\%)\end{array}$} & MrCons & MsCons & \multirow{2}{*}{$\begin{array}{l}\text { total } \\
(\%)\end{array}$} \\
\hline & $\begin{array}{l}n=3 \\
(6.25 \%)\end{array}$ & $\begin{array}{l}\mathrm{n}=45 \\
(93.75 \%)\end{array}$ & & $\begin{array}{l}\mathrm{n}=26 \\
(15.12 \%)\end{array}$ & $\begin{array}{l}\mathrm{n}=146 \\
(84.88 \%)\end{array}$ & \\
\hline $\begin{array}{l}\text { Erythromycin } \\
\text { resistant }\end{array}$ & $1(33.3)$ & $13(28.9)$ & $14(29.2)$ & $21(80.8)$ & $44(30.1)$ & $65(37.8)$ \\
\hline $\begin{array}{l}\text { Clindamycin } \\
\text { Resistant }\end{array}$ & $2(66.7)$ & $6(13.3)$ & $8(16.7)$ & $14(53.8)$ & $34(23.3)$ & $48(27.9)$ \\
\hline MLSBi & $1(33.3)$ & $2(4.4)$ & $3(6.3)$ & $1(3.8)$ & $6(4.1)$ & $7(4.1)$ \\
\hline MLSBc & $01(33.3)$ & $5(11.1)$ & $6(12.5)$ & $10(38.5)$ & $26(17.8)$ & $36(20.9)$ \\
\hline MS & $1(33.3)$ & $8(17.8)$ & $9(18.8)$ & $11(42.3)$ & $18(12.3)$ & $29(16.9)$ \\
\hline \multicolumn{7}{|c|}{ MSSA-Methicillin sensitive S. aureus } \\
\hline
\end{tabular}

Fig.1 Shows graphical presentation of antimicrobial resistance percentage of nasal carriage MRSA and MSSA

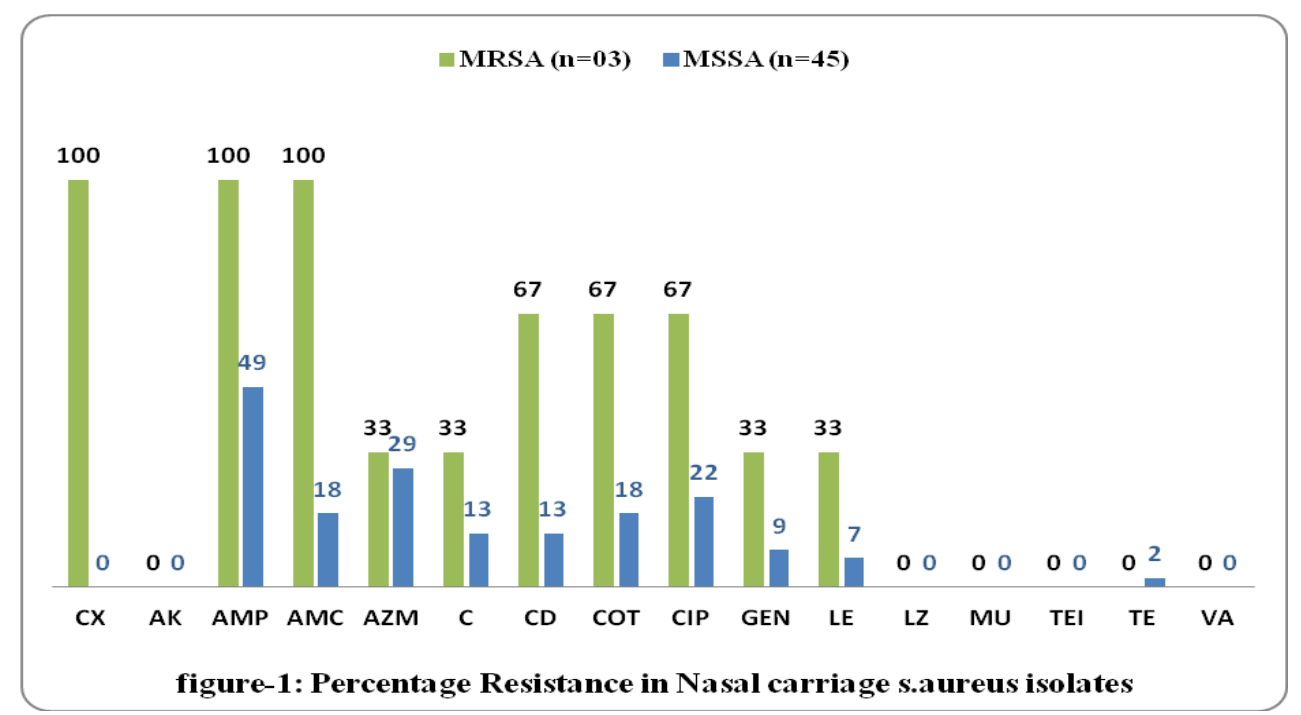


Among the Staphylococcal isolates, 3 (6.25\%) were MRSA, 45 (93.75\%) were MSSA (Methicillin sensitive S. aureus), 26 (15.12\%) were MRCoNS (methicillin resistant CoNS) and 146 (84.88\%) were MSCoNS (Methicillin sensitive CoNS). Shiv Sekhar Chatter Jee et al., (2009) also found similar MRSA $3.89 \%$ in Chandigarh while $16.3 \%$ was observed by Ashish pathak et al., (2010) in Ujjain (MP). Yukti Sharma et al., (2014) also found Staphylococcus aureus colonisation to be $12 \%$. MRSA was identified in $5 \%$ of total isolates. Staphylococcus aureus colonization was found to be $12 \%$. Erythromycin resistance was seen in $14(29.29 \%)$ isolates while 8 (16.7) isolates found to be resistant to Clindamycin among $S$. aureus isolates. Among these isolates Inducible clindamycin was seen in $3(6.3 \%)$ isolates of $S$. aureus. Constitutive MLSB phenotype (MLSBc) and the MS phenotype was seen in $6(12.5 \%)$ and $9(18.75 \%)$ isolates among $S$. aureus respectively. The percentage of inducible clindamycin resistance was higher among Methicillin-resistant S. aureus (MRSA) (33.3 $\%)$ compared to methicillin-sensitive $S$. aureus (4.4\%). It was observed that Induiciable Clindamycin Resistance (MLSBi) isolates were significantly more among the MRSA than the MSSA isolates.

Similarly, erythromycin resistance was seen in $65(37.79 \%)$ of the CoNS isolates. Among these isolates Induciable clindamycin resistance (MLSBi) was seen in $7(4.07 \%)$ isolates. A total 36 (20.9\%) and 29(16.86\%) isolates showed Constitutive MLSB phenotype (MLSBc) and MS phenotype isolates respectively among CONS. Baragundi Mahesh et al., (2013) found inducible Clindamycin resistance as $24.44 \%$, $12.04 \%, 16.39 \%$, and $3.22 \%$ in the MRSA, MSSA, MRCoNS and the MSCoNS nasal isolates respectively. Similarly, Pal et al., (2010) found inducible Clindamycin resistance $6.93 \%$ in MSSA and $6 \%$ in
MSCoNS. The prevalence of the S.aureus nasal carriage was higher among the male (27; 56.25\%) than among the female (21; $43.75 \%$ ). Sharon et al., (2010) found the same as in $54 \%$ male and $45.7 \%$ in female. Similarly Shakya et al., (2010) found the similar as $42 \%$ and $57 \%$ in male and female respectively. While contrast finding was also observed by Ahmad S et al., (2010) as 22.5\% and $44.6 \%$ in male and female respectively.

The similar study by other Indian scholars on clinical isolates of $S$. aureus showed MLSBi prevalence of $14.5 \%$ and $13.1 \%$. Results of present study vary a little compared to other Indian studies that reported MLSBi prevalence of $27.6 \%$ and $38.4 \%$ among MRSA, and $1.6 \%$ and $12.9 \%$ among MSSA. Association of methicillin resistance along with the presence of the MLSBi phenotype was found to be statistically significant in our study. Dhivya Lakshmi and Chitralekha Saikumar (2018) observed $38.3 \%$ erythromycin resistance and $15.5 \%$ clindamycin resistance in the similar study done in India. He observed that MLSBi and MS phenotype in MRSA were $28.5 \%$ and $48.2 \%$ respectively while $4.8 \%$ and $8.8 \%$ for MSSA. The Constitutive MLSBc resistance was seen in $10.7 \%$ of MRSA isolates and $2.4 \%$ in MSSA. Deotale et al., (2010) found it in $7.3 \%$ of MRSA isolates. The Similar studies (Prabhu Kavitha, 2017; Deotale et al., 2010) reported erythromycin resistance to be $28.42 \%$ and $32.4 \%$ respectively.

All the Staphylococcus aureus isolates were sensitive to Amikacin, mupiriocin, teicoplanin, vancomycin and linezolid (100\%). Ampicillin was the most resistant (54\%) antibiotic followed by Erythromycin $(40 \%)$, ciprofloxacin (33\%), cotrimoxazole (31\%), Ampicillin calvulanic acid (23\%), clindamycin (17\%) and chloramphenicol (15\%), gentamycin (10\%), levofloxacin $(8 \%)$ and doxycycline (2\%). Methicillin resistance 
was seen in $6.25 \%$ of the $S$. aureus isolates, both by the disc diffusion test and by the Oxacillin Screen Agar (OSA) test. Yukti Sharma et al., (2014) found that Fluoroquinolone was remarkable resistance against Staphylococcus aureus whereas all strains were sensitive to vancomycin, teicoplanin, linezolid, quinupristindalfopristin.

Anterior naris is the ecological niches of the $S$. aureus which make the organism a potent agent for nosocomial infection as well as community associated staphylococcal infection (Lowy FD, 1998). Thus, the prevalence of inducible clindamycin resistance in $S$. aureus isolated from anterior nasal cavity was evaluated to justify and rule out the hazardous role of carriage in the community and to provide a therapeutic guideline for better patient management. An accurate susceptibility data is a key factor for appropriate therapy decisions. The sensitivity of the D test was found $100 \%$ when it was correlated with the detection of the erm and the $m s r$ genes by PCR.

D test can be used as a simple and reliable method for detrcting the inducible and the constitutive $\mathrm{CL}$ resistance in the routine clinical laboratories. Without proper classification of the isolates with inducible clindamycin resistance would lead to treatment failures. Thus, the therapeutic use of clind clindamycin can be omitted in the patients with infections caused by the inducible resistant strains and the therapeutic failures may thus be avoided.

\section{References}

Ahmad S. Prevalence of Staphylococcus aureus colonization among healthcare workers at a specialist hospital in Saudi Arabia. Journal of Clinical and
Diagnostic Research. 2010; (4): 243841.

Ashish Pathak, Yogyata Marothi, Rama V Iyer, Binita Singh, Megha Sharma, Bo Eriksson, Ragini Macaden,; Nasal Carriage and Antimicrobial Susceptibility of Staphylococcus aureus in healthy preschool children in Ujjain, India; BMC Pediatrics 2010, 10:100

Baird D. Staphylococcus: cluster-forming Gram-positive cocci. In: Collee JG, Marmion BP, Fraser AG, Simmons A, editors. Mackie and McCartney practical medical microbiology. 14th ed. New Delhi: Elsevier; 2016. p. 255.

Baragundi Mahesh C., KulKarni raMaKant B., sataraddi Jagadeesh V, (The Prevalence of Inducible and Constitutive Clindamycin Resistance Among the Nasal Isolates of Staphylococci Journal of Clinical and Diagnostic Research. 2013 Aug, Vol7(8): 1620-1622).

Ciraj AM, Vinod P, Sreejith G, Rajani K. Inducible clindamycin resistance among clinical isolates of staphylococci. Indian J Path Microbiol. 2009; 52: 49-51. [PubMed: 19136780]

CLSI document M100-S17. Wayne Pa: CLSI; 2017. Clinical and laboratory standards institute. Performance standards for antimicrobial susceptibility testing; seventeenth informational supplement.

Deotale V, Mendiratta DK, Raut U, Narang P. Inducible clindamycin resistance in Staphylococcus aureus isolated from clinical samples. Indian

Dhivya Lakshmi, S., and Chitralekha Saikumar; Detection of Inducible Clindamycin Resistance with Erythromycin in Clinical Isolates and Its Prevalence among Methicillin Resistant Staphylococcus aureus; Int.J.Curr.Microbiol.App.Sci (2018) 7(1): 2405-2409. 
Drinkovic D, Fuller ER, Shore KP, Holland DJ, Ellis-Pegler R. Clindamycin treatment of the Staphylococcus aureus which expressed inducible clindamycin resistance. J Antimicrob Chemother. 2001; 48: 315-16.

Fahriye E, Efgan DG, Aysen B, Tekin K. Determination of antimicrobial susceptibility patterns and inducible clindamycin resistance in Staphylococcus aureus strains recovered from southeastern Turkey. J Microbiol, Immunol, and Infect. 2011; 44: 57-62.

Fiebelkorn KR, Crawford SA, McElmeel ML, Jorgensen JH. Practical disk diffusion method for detection of inducible clindamycin resistance in Staphylococcus aureus and coagulasenegative staphylococci. J Clin Microbiol. 2003; 41: 4740-4.

Kloos WE, Banerman TL. Staphylococcus and Micrococcus, Chapter 22. In: Manual of Clinical Microbiology. 7th ed. Murray PR, Baron EJ, Pfaller MA, Tenoer FC, Yolken RH, editors. Washington DC. ASM Press. 1999; 264-82.

Lowy FD. Staphylococcus aureus infections. N Engl J Med. 1998; 339: 520-32.

Pal N, Sharma B, Sharma R, Vyas L. Detection of inducible clindamycin resistance among Staphylococcal isolates from different clinical specimens in western India. J Posgrad Med. 2010; 56 (3): 182-85.

Prabhu Kavitha, Sunil Rao, and Venkatakrishna Rao. Inducible Clindamycin Resistance in Staphylococcus aureus Isolated from Clinical Samples. Journal of Laboratory Physicians 3.1 (2011): 25-27. PMC. Web. 23 Sept. 2017.

Roberts MC, Sutcliffe J, Courvalin P, Jensen LB, Rude. J, Seppala H. The nomenclature for the macrolide lincosamide streptogramin B resistance determinants. Antimicrob Agents Chemother. 1999; 43(12):2823-30.

Schreckenberger PC, Ilendo E, Ristow KL. Incidence of constitutive and inducible clindamycin resistance in Staphylococcus aureus and coagulasenegative staphylococci in a community and a tertiary care hospital. J Clin Microbiol. 2004; 42: 2777-9.

Shakya B, Shrestha S, Mitra T. Nasal carriage rate of methicillin resistant Staphylococcus aureus among at National Medical College Teaching Hospital, Birgunj, Nepal. Nepal Med Coll J. 2010; 12(1): 26-29.

Sharon Rainy Rongpharpi et al., Nasal Carriage of Staphylococcus aureus, Journal of Clinical and Diagnostic Research. 2013 February, Vol-7(2): 257-260

Shiv Sekhar Chatterjee, Pallab Ray, Arun Aggarwal, Anindita Das and Meera Sharma; Indian J Med Res 130, December 2009, pp 742-748; A community-based study on nasal carriage of Staphylococcus aureus.

Shobha KL, Rao PS, Thomas J. Survey of Staphylococcus isolates among hospital personnel, environment and their antibiogram with special emphasis on methicillin resistance. Indian $\mathrm{J}$ Med Microbiol. 2005; 23(3):186-88.

Siberry GK, Tekle T, Carroll K, Dick J. Failure of the clindamycin treatment of the methicillin resistant Staphylococcus aureus which expressed inducible clindamycin resistance in vitro. Clin Infect Dis. 2003; 37, 1257-60.

The Clinical and Laboratory Standards Institute (CLSI) (2017) Performance standards for antimicrobial susceptibility testing: eighteenth informational Supplement, M100-S18 CLSI. 
Vinodhkumaradithyaa A, Uma A, Srinivasan M, Ananthalakshmi I. Nasal carriage of Methicillin -Resistant Staphylococcus aureus among surgical unit staff. Institute of Madurai Medical College, Madurai. Jpn J Infect Dis. 2009; 62: 228-29.

Weisblum B, Demohn V. Erythromycininducible resistance in Staphylococcus aureus: a survey on the antibiotic classes which were involved. J. Bacteriol. 1969; 98: 447-52.

Weisblum B. Erythromycin resistance due to ribosome modification. Antimicrob. Agents Chemother. 1995; 39: 577-85.
Winn WC, Jr, Koneman EW, Allen SD, Procop GW, Janda WM, Schreckenberger PC, et al., 6th ed. Baltimore (USA): Lippincott Williams and Wilkins; 2016. Koneman's color atlas and textbook of diagnostic microbiology.

Yukti Sharma, Sanjay Jain, Harshvardhan Singh, and Vasudha Govil; Staphylococcus aureus: Screening for Nasal Carriers in a community Setting with Special Reference to MRSA; Scientifica; Volume 2014 (2014), Article ID 479048, 5 pages.

\section{How to cite this article:}

Manish Kumar Diwakar, Ankur Goyal, Santosh Verma and Neha Srivastava. 2018. Prevalence of Inducible Clindamycin Resistance among Nasal Carriage Staphylococcus aureus among Healthy Population. Int.J.Curr.Microbiol.App.Sci. 7(05): 2509-2517.

doi: https://doi.org/10.20546/ijcmas.2018.705.289 